\title{
Design and Experimental Validation of an Odometric and Goniometric Localization System for Outdoor Robot Vehicles
}

\author{
Ph. Bonnifait and G. Garcia \\ Institut de Recherche en Cybernétique de Nantes UMR 6597 \\ Ecole Centrale de Nantes \\ 1 rue de la Noë, BP 92101, 44321 Nantes cedex 03, France; fax: (33) 2-40-37-25-22 \\ e-mail: Philippe.Bonnifait@lan.ec-nantes.fr - Gaetan.Garcia@lan.ec-nantes.fr
}

\begin{abstract}
A 2D mobile robot localization system which uses odometry and the azimuth angles of known landmarks is presented. Observability analysis helps to determine situations where such a system may undergo difficulties, and gives information on its behavior when one of the beacons is hidden. Experimental results are presented.
\end{abstract}

Keywords: outdoor mobile robot; dynamic localization; optical triangulation system; multisensor integration; non-linear observability; Kalman filtering.

\section{INTRODUCTION}

Real-time localization is a fundamental requirement of autonomous mobile robots. In this paper, we present a localization system which is intended for later use in the context of Computer-Integrated Road Construction (CIRC).

Today, dedicated CAD systems are used extensively to define precisely the various layers that constitute the pavement, with respect to an absolute reference frame. For lack of a convenient localization system for the machines, a keystone to automation, the CAD data cannot be directly fed to the machines for controlling their paths and tools.

Localization can be broadly separated into two distinct approaches: dead reckoning and absolute reckoning. Starting from a known position and orientation and by integrating elementary displacements, dead reckoning continuously provides location data with no delay and without any knowledge of the environment. Nevertheless, it generally requires an initial absolute estimated location. Moreover, the integration of the noisy displacements causes boundless drift of the estimation. Absolute localization refers to navigation with respect to a coordinate frame based on the environment. A popular solution belonging to 
this category is GPS. The recent advances in differential GPS seem to provide enough accuracy for a significant number of applications [1]. Nevertheless, when some satellites are obscured by trees, mountains, bridges or buildings for instance, the precision can decrease so much that another localization system has to be used. Considering the current state-of-the-art of technology [2], it is clear that, in the case of our application, we must turn ourselves to optical systems using active beacons in order to achieve the precision we are looking for (a few centimeters in position).

Absolute and relative localization are complementary. Generally, absolute systems do not provide localization data at a high frequency, whereas relative systems can. Moreover, if an absolute system does not detect enough landmarks for its localization process, relative techniques can calculate estimates during limited periods of time. Finally, combinations of the two approaches can yield very accurate positioning systems. Our approach falls into this type. We use an optical triangulation system which measures azimuth angles of known beacons to correct odometric estimations. The most common formalism used to associate these two kinds of systems is the Kalman filter, as exemplified by the localization systems of $[1,3,4,5]$.

The paper is organized as follows. Part two is devoted to the presentation of the triangulation sensor. The localization algorithm (which will be called "MLS" - Multisensor Localization System) and its Kalman formalism are presented in part three. Section four is devoted to the study of the observability of the posture of the robot depending on the number of beacons that can be detected and on the movement of the vehicle. Finally, part five reports the results of real outdoor experiments that show that our system reaches a precision of a few centimeters on large evolution fields. Our tests have been performed by moving along paths, not just checking endpoints in a static posture, as is often done.

The main contributions of the paper lie in the study of the observability of the non-linear system and in the implementation and validation of the MLS in real time.

\section{THE OPTICAL SENSOR AND ITS SETUP ON A VEHICLE}

The artificial beacons we use are battery-powered twin light sources which are switched on and off using an HF link in order to decrease energy consumption. The sensor is a linear CCD camera which rotates with a constant speed $(\approx 1 \mathrm{rad} / \mathrm{s})$ around an axis fixed to the vehicle. Hence, the landmarks are detected one at a time and intermittently. The rotation axis passes through the optical center (figure 1). The azimuth angles (denoted $\lambda$ ) are measured with respect to the heading by an optical encoder. The accuracy 
of this measurement depends on the scanning frequency of the camera and on the rotation speed. The low speed we have chosen provides accurate angles (the standard deviation of an azimuth angle is $2.210^{-3}$ $\mathrm{rad})$.

The sensor is also able to measure simultaneously an elevation angle (see figure 1). This angle is not used here, but has previously been used for deterministic 3D posture calculation.
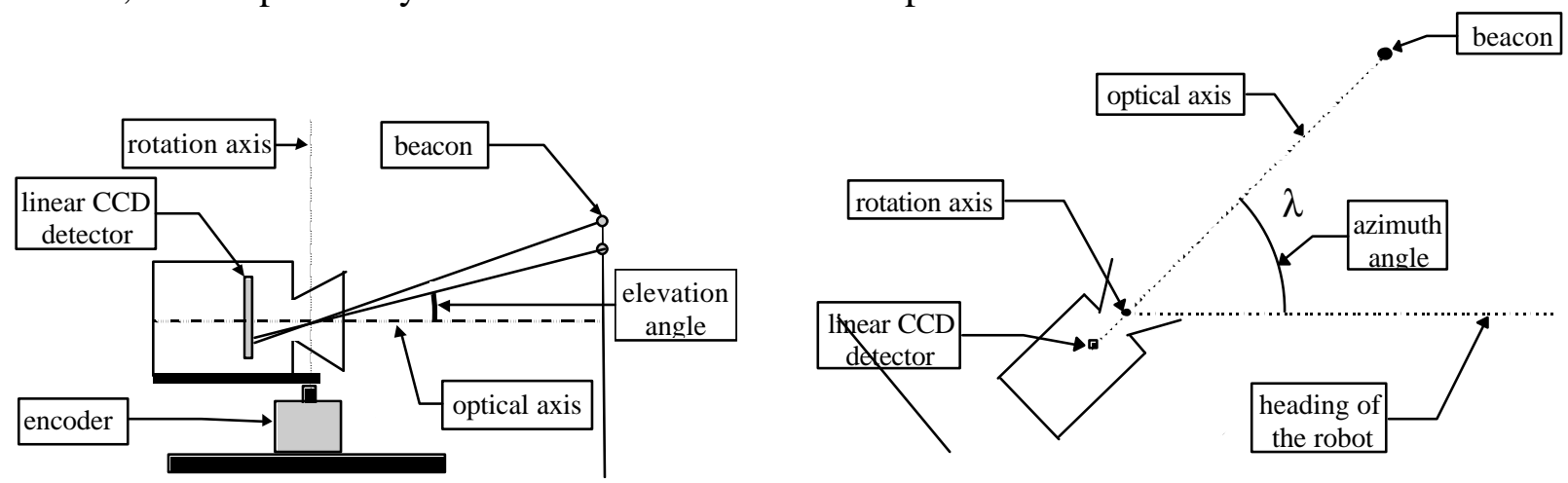

figure 1: side and top views of the azimuth sensor.

A particular problem of this kind of sensor is that it provides asynchronous measurements. Asynchronous means that the time of the detection of a beacon cannot be known beforehand, since the angular interval between two beacons depends on the position and movement of the mobile.

There is no constraint on the position of the camera with respect to the odometric reference point. It means that the projections on the $X Y$ plane of the middle of the wheel base (denoted $M$ ) and of the rotation axis of the camera $(S)$ can be different (figure 2). This is essential in practice, since the sensor may not fit above point $M$ on a given machine.

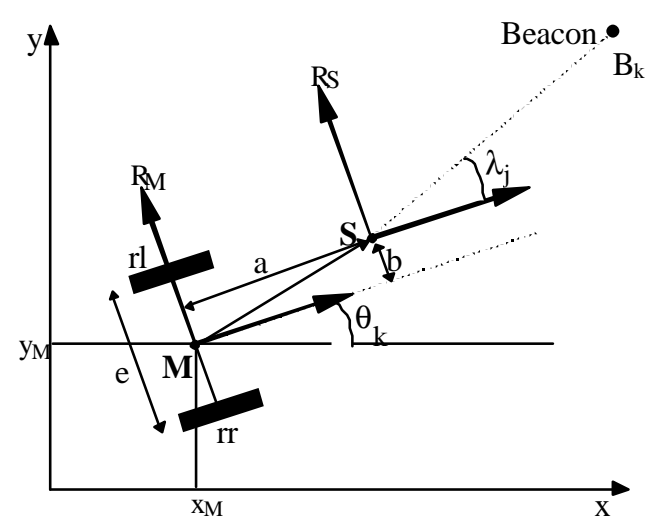

figure 2: model of the vehicle.

The rotation between frames $R_{M}$ and $R_{S}$ can be set to null by an appropriate calibration procedure, so that the transform between the two frames reduces to a translation vector $(a, b)$. 


\section{THE MLS ALGORITHM}

The algorithm presented below relies on an odometric prediction of the posture between the time instants of the readings of two azimuth angles, and uses these angles to update the posture. This multisensor approach takes advantage of sensor redundancy and complementarity $[3,5,6]$.

\section{A. Evolution model for point $S$}

In order to take into account the translation between frames $R_{M}$ and $R_{S}$ (see figure 2), we need to express the evolution of frame $\mathrm{R}_{\mathrm{S}}$ as a function of the elementary rotations of the wheels, $\Delta q r_{i}$ and $\Delta q l_{i}$, between time instants $i$ and $i-1$, provided by the optical encoders attached to the wheels.

$$
\Delta q_{i}=\left[\begin{array}{ll}
\Delta q r_{i} & \Delta q l_{i}
\end{array}\right]^{T}
$$

Let $r r$ and $r l$ denote the radii of the wheels, which can be a bit different from one another for tire wheels. The wheel base $e$ represents the distance between the middle of the two tires. First, we can compute $\Delta D_{i}$, the distance traveled by point $M$, and $\Delta \theta_{i}$ the elementary rotation of $R_{S}$ and $R_{M}$ :

$$
\Delta D_{i}=\frac{r r \cdot \Delta q r_{i}+r l \cdot \Delta q l_{i}}{2} \Delta \theta_{i}=\frac{r r \cdot \Delta q r_{i}-r l \cdot \Delta q l_{i}}{e}
$$

Let the vector $X_{i}=\left[\begin{array}{lll}x_{i} & y_{i} & \theta_{i}\end{array}\right]^{T}$ represent the posture of $R_{S}$. By considering the path as circular between time indexes $i$ and $i+1$, and by taking into account the translation, we obtain [7]:

$$
\left\{\begin{array}{l}
x_{i+1}=x_{i}+\Delta D_{i} \cdot \cos \left(\theta_{i}+\Delta \theta_{i} / 2\right)-\Delta \theta_{i} \cdot\left(a \cdot \sin \theta_{i}+b \cdot \cos \theta_{i}\right) \\
y_{i+1}=y_{i}+\Delta D_{i} \cdot \sin \left(\theta_{i}+\Delta \theta_{i} / 2\right)+\Delta \theta_{i} \cdot\left(a \cdot \cos \theta_{i}-b \cdot \sin \theta_{i}\right) \\
\theta_{i+1}=\theta_{i}+\Delta \theta
\end{array}\right.
$$

Using equation (2), this system can be rewritten as:

$$
X_{i+1}=F\left(X_{i}, \Delta q_{i}\right)
$$

The plant model we obtain is a good approximation of reality when $\Delta \theta_{i}$ and $\Delta D_{i}$ are small, which requires that the sampling period be adapted to the linear and angular velocities of the vehicle.

\section{B. State-space description}

The observations are the azimuth angles $\lambda_{j}$ of the landmarks $B_{k}$, the coordinates of which are denoted $\left(\mathrm{x}_{\mathrm{B}^{\mathrm{k}}}, \mathrm{y}_{\mathrm{B}^{\mathrm{k}}}\right)$. The observation equation is scalar, but non-stationary since it depends on the coordinates of the landmark detected.

Suppose this measurement occurs at time instant $j$; figure 2 yields: 


$$
\lambda_{j}=\operatorname{atan} 2\left(y_{B^{k}}-y_{j}, x_{B^{k}}-x_{j}\right)-\theta_{j}=g^{k}\left(X_{j}\right)
$$

Equations (1), (4) and (5) are actually corrupted by noises which are supposed to be zero-mean, independent and white. The system obtained is non-linear and its state-space description is:

$$
\left\{\begin{array}{l}
X_{i+1}=F\left(X_{i}, \Delta q_{i}\right)+\alpha_{i} \\
\Delta q_{i}=\left(\Delta q_{i}\right)_{\text {real }}+\gamma_{i} \\
\lambda_{j}=g^{k}\left(X_{j}\right)+\beta_{j}
\end{array}\right.
$$

$\alpha_{\mathrm{i}}$ is the model noise, which represents the effects of slippage or skid on the ground plus the effects of errors on robot parameters such as $r r, r l$ and $e . \gamma_{i}$ and $\beta_{j}$ are white noises due to measurement errors. The covariance matrices of these noises are denoted by $Q_{\alpha}, Q_{\gamma}$ and $Q_{\beta}$.

One can notice that the state-space description has two time indexes $i$ and $j$. The last one indicates that the goniometric measurements are done asynchronously and intermittently.

In practice, the state vector also contains the radii of the rubber tire wheels, because they are subject to slow variations over time. Simulations reported in [8] prove that the filter is able to compensate for initial errors on the radii and to track slow variations. This avoids the bias to which the output location is subject when the radii are not identified.

\section{Kalman filtering formulation}

Because the system (6) is non-linear, we use an Extended Kalman Filter (EKF) to estimate the state $X$. The equations are linearized thanks to the Taylor series theorem. This is usually a good approximation, if the errors are small enough and the non linearities are benign so that the higher order terms can be neglected. There are few theoretical results to indicate when such a design will be successful. La Scala et al. [9] derive sufficient conditions to ensure that the errors of the EKF will remain bounded, but their results are established for a linear observation equation. As the convergence of an EKF cannot be demonstrated, we will propose in section IV to detect problematic situations thanks to the study of the observability of the non-linear system.

The MLS algorithm works in two steps: prediction and filtering. In the sequel, $\hat{X}_{j / k}$ represents the estimated $X$ at time index $j$ using all the information available at time $k$.

\section{Step 1: prediction}


Between two absolute readings, a "high" frequency $(20 \mathrm{~Hz}$, as compared to the frequency of the absolute readings $\approx 0.5 \mathrm{~Hz}$ ) state and error prediction phase occurs.

$$
\hat{X}_{i+1 / i}=F\left(\hat{X}_{i / i}, \Delta q_{i}\right)
$$

Since the estimation error is supposed to be sufficiently small and the random vectors $X$ and $\Delta q$ are clearly independent, the covariance matrix $P$ of the estimation error is given by:

$$
P_{i+1 / i} \approx A_{i} \cdot P_{i / i} \cdot A_{i}^{t}+B_{i} \cdot Q_{\gamma} \cdot B_{i}^{t}+Q_{\alpha}
$$

$A_{i}$ and $B_{i}$ are the Jacobian matrices of $F$ with respect to $X$ and $\Delta q$.

\section{Step 2: filtering}

The external measure, available at time instant $j$, is taken into account at the next odometric instant. This is acceptable because the change in robot posture between two odometric instants $(<5 \mathrm{~mm})$ is negligible with respect to the desired precision. We use the Mahalanobis distance in order to check if the measured angle corresponds to a real beacon. Suppose that we know that beacon $k$ is supposed to be detected. Then, the Mahalanobis distance is given by [7]:

$$
D_{j}^{k}=\left(\lambda_{j}-g^{k}\left(\hat{X}_{j+1 / j}\right)\right)^{2} \cdot\left(C_{j} \cdot P_{j+1 / j} \cdot C_{j}^{t}+Q_{\beta}\right)^{-1} \text { and } C_{j}=\left[\partial g^{k} / \partial X\right]_{\hat{X}_{j+l / j}}
$$

If $D_{j}^{k}$ is lower than a predefined threshold, the measure and the prediction are considered as coherent, and we correct the predicted state using expressions (10) - (12). Otherwise, no correction is performed. In such a situation, it is impossible to know whether the odometric estimate is wrong (slippage etc.) or if the measure is erroneous (beacon reflection etc.). The strategy we have chosen is: if the measures of the three beacons become incoherent or if one beacon is incoherent for more than three times, we consider that the MLS has to be reinitialized and we stop the robot.

$$
\hat{X}_{j+1 / j+1}=\hat{X}_{j+1 / j}+K_{j} \cdot\left(\lambda_{j}-g^{k}\left(\hat{X}_{j+1 / j}\right)\right)
$$

The Kalman gain vector and the covariance matrix are given by:

$$
\begin{gathered}
K_{j}=P_{j+1 / j} \cdot C_{j}^{t} \cdot\left(C_{j} \cdot P_{j+1 / j} \cdot C_{j}^{t}+Q_{\beta}\right)^{-1} \\
P_{j+1 / j+1}=\left(I_{33}-K_{j} \cdot C_{j}\right) \cdot P_{j+1 / j}
\end{gathered}
$$




\section{SYSTEM OBSERVABILITY}

The process of designing a localizer for a mobile vehicle, especially when using both relative and absolute sensors, can be seen as equivalent to designing a (generally non-linear) observer of the state of the vehicle. The study of system observability allows us to determine situations in which any observer may (or sometimes will) undergo problems [10]. In practice, these situations are not always easy to determine intuitively, and extensive simulation tests, defined more or less randomly, may not detect these situations. The sensitivity of the observer to obscured beacons is also investigated through this observability analysis. Nevertheless, it does not give any information about the accuracy of the observer.

It should be noted that, in this section, we use a continuous model of our system, in order to be able to apply the standard tools of non-linear observability. Moreover, we consider a robot where $S=M$ (figure 2). This hypothesis simplifies the calculations.

\section{A. Non-linear observability concept}

For non-linear systems like the one of equation (13), the observability of the state depends on the inputs, contrary to the linear case.

$$
\begin{cases}\dot{X}=f(X, U) & X \in \mathfrak{R}^{n} \\ \Lambda=g(X) & \Lambda \in \mathfrak{R}^{p}\end{cases}
$$

This system is observable if, and only if, the state can be expressed as a function of the observation $\Lambda$, the input, and their derivatives with respect to time:

$$
X=\varphi\left(\Lambda, \dot{\Lambda}, \ldots, \Lambda^{\left(k_{1}\right)}, U, \dot{U}, \ldots, U^{\left(k_{2}\right)}\right)
$$

A sufficient condition allows one to conclude to the observability of a system, by computing the rank of an observability nx(n.p) matrix $\boldsymbol{O}[11]$.

$$
\boldsymbol{O}=\left[\begin{array}{lllllll}
d g_{1} & d L_{f} g_{1} & \ldots d L_{f}^{n-1} g_{1} & \ldots & d g_{p} & \ldots & d L_{f}^{n-1} g_{p}
\end{array}\right]
$$

- $d h$ is the gradient vector of $h$ with respect to the state $X$.

- $L_{f} g$ is the Lie derivative of $g$ with respect to $f: L_{f} g=(\partial g / \partial x) . f=\dot{\Lambda}$

The sufficient condition is: If $\operatorname{rank}(\boldsymbol{O})=n$, then the system is observable.

Yet, if the rank of $\boldsymbol{O}$ is not full, non-observability is not proved, contrary to the linear case. 


\section{B. Continuous system modeling}

The system we consider is composed of the robot, the goniometric sensor and the beacons. Let $\mathrm{U}=\left[\mathrm{u}_{1}, \mathrm{u}_{2}\right]^{\mathrm{t}}$ be the control vector, where $\mathrm{u}_{1}$ and $\mathrm{u}_{2}$ are the linear and angular velocities. We have:

$$
\left\{\begin{array}{l}
\dot{x}=u_{1} \cdot \cos \theta \\
\dot{y}=u_{1} \cdot \sin \theta \\
\dot{\theta}=u_{2}
\end{array} \Leftrightarrow\{\dot{X}=f(X, U)\right.
$$

If we suppose that the azimuth angles of the three landmarks are continuously available, we concatenate them in an observation vector $\Lambda$ (see equation (5)).

$$
\Lambda=\left[\begin{array}{c}
\lambda^{1} \\
\cdots \\
\lambda^{3}
\end{array}\right]=\left[\begin{array}{c}
\operatorname{atan} 2\left(\mathrm{y}_{\mathrm{B}^{1}}-\mathrm{y}, \mathrm{x}_{\mathrm{B}^{1}}-\mathrm{x}\right)-\theta \\
\cdots \\
\operatorname{atan} 2\left(\mathrm{y}_{\mathrm{B}^{3}}-\mathrm{y}, \mathrm{x}_{\mathrm{B}^{3}}-\mathrm{x}\right)-\theta
\end{array}\right]=\mathrm{g}(\mathrm{X})
$$

Nevertheless, while the vehicle is moving, one or even two landmarks can be hidden or too far from the sensor to be detected. In such a case, the dimension of the output vector $\Lambda$ decreases to a value which will be denoted $p$.

With one beacon $(p=1)$, the observation is a scalar. The observation matrix is square. After calculation, we find that $\operatorname{det}(\boldsymbol{O})$ is always equal to zero, whatever the state and the input. In this case, the system is generically non-observable.

\section{Analysis with three beacons}

\section{Observability without taking into account the inputs}

In this part $p=3$, which is the normal situation for our localizer. In such a case, the determination of the state $X$, given the three outputs $\lambda_{i}$, involves inverting $g$. Let us consider $\boldsymbol{O}_{1}$ a sub-matrix of the observation matrix. $\boldsymbol{O}_{\boldsymbol{I}}$ does not depend on the input:

$$
\boldsymbol{O}_{1}=\left[\begin{array}{lll}
d g^{1} & d g^{2} & d g^{3}
\end{array}\right]
$$

If $\operatorname{det}\left(\boldsymbol{O}_{l}\right)$ is not equal to zero, nor is $\operatorname{det}(\boldsymbol{O})$. The curve $\operatorname{det}\left(\boldsymbol{O}_{l}\right)=0$ is the circle (called $C$ ) defined by the three beacons.

The difficulty for this case can be interpreted geometrically. A solution for determining the location of the mobile given the outputs only, is to use the fact that two measures, $\lambda_{i}$ and $\lambda_{j}$, constrain the robot to lie on a given circle defined by beacons $B_{i}$ and $B_{j}$ and the angle $\left(\lambda_{i}-\lambda_{j}\right)$. The three circles available intersect at 
the location of the robot. Here, the three circles merge in $C$ and so $X$ cannot be determined by the geometrical solution.

Since $\operatorname{rank}(\boldsymbol{O})=3$ anywhere except on $C$, we can conclude that the system is observable anywhere except, perhaps, on $C$. Since outside $C$, observability does not depend on the input, the system is said to be uniformly observable.

\section{Study of the degenerate situation}

By factoring $u_{1}$ and $u_{2}$ in the Lie derivatives $L_{f} g(X, \Lambda)$, it is easy to prove that, on $C$, the rank is at most 2 , if the vehicle is motionless. Since the rank condition is only sufficient, we cannot assert that the system is not observable. This is what we call a "problematic situation". When observability cannot be proved, the behavior of the observer shall be checked through simulations.

In the following simulations, we compare the estimations of a filter with no initial error, and the same one, running on the same noisy measures, but with an erroneous initial estimate (with position and heading errors). These simulations are performed with Gaussian distribution errors.

Simulation $A$ of figure 3 shows that, for a motionless vehicle on $C$, our observer is not able to drive an initial estimation error to zero. Yet, in all the simulations we performed, the estimation error remained bounded.
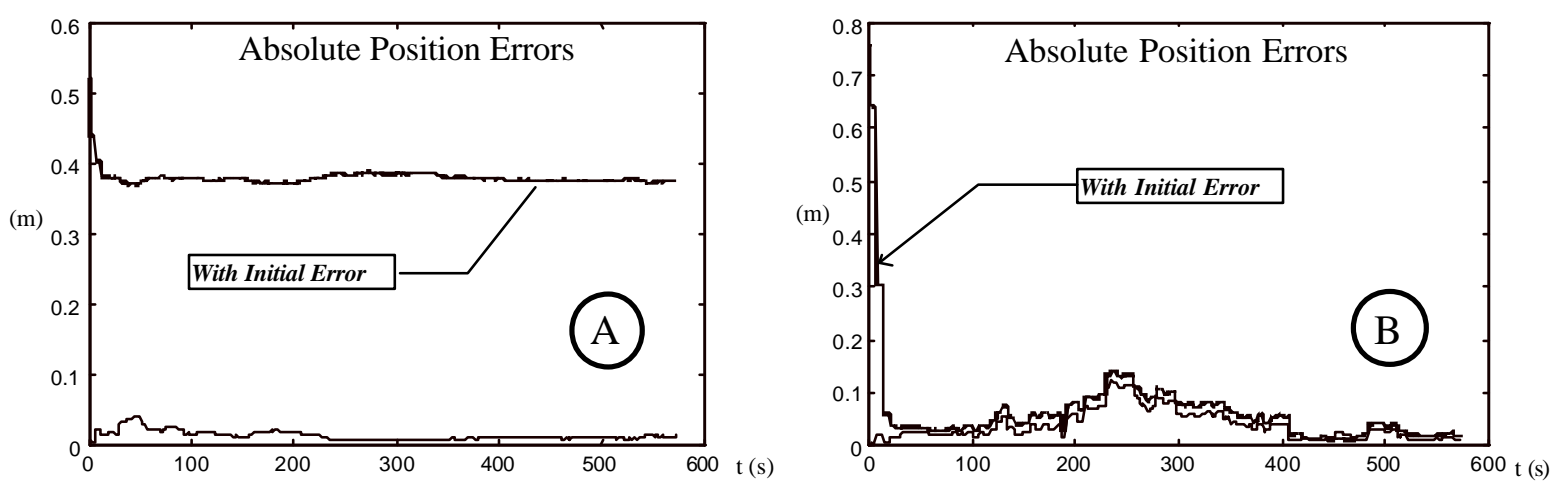

\section{figure 3: filter errors for a motionless vehicle on $C$ (test $A$ ) and for a vehicle tracking an arc of $C$ (test B)}

Let us now consider the case when the robot moves on $C$. We could calculate higher order derivatives $\left(d L_{f} g^{\mathrm{i}}\right.$ and $d L_{f}^{2} g^{i}$ ) but the calculations become quickly untractable. Instead, we choose to test our observer by simulating a mobile running on this possibly difficult trajectory. The results of the test $B$ of figure 3 show that a filter with initial error converges quickly and its outputs become identical to the outputs of a filter with no initial error running on the same data. This kind of behavior indicates the convergence of the filter. 


\section{Analysis with two beacons}

In this part, we suppose that the sensor only detects two landmarks. We have $p=2$. Therefore, state observation cannot be obtained using the outputs only. The evolution model and the input are of crucial importance here.

If the vehicle is motionless, it is easy to show, as in C.2, that the rank of $\mathbf{O}$ is at most 2. Intuitively, with two beacons and when the vehicle is motionless, it is clear that the filter cannot estimate the three independent parameters.

In order to test the rank of $\boldsymbol{O}$, we have computed the determinants of the $C_{6}^{3}=20$ sub-matrices of $\boldsymbol{O}$ thanks to the symbolic computation software "Maple" [10]. Finally, the problematic situations obtained are when the robot is tracking one of the lines of figure 4, whatever its linear speed.

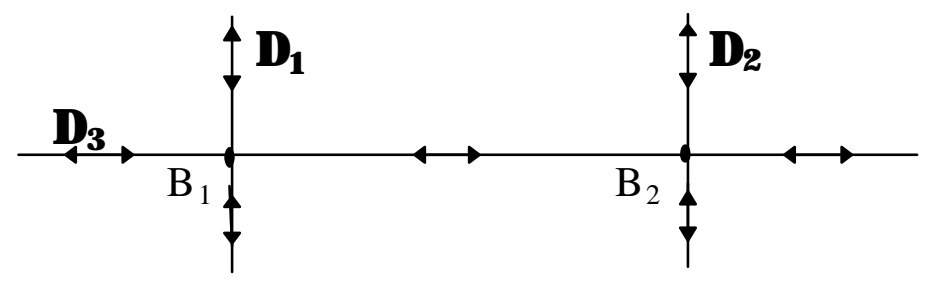

figure 4: problematic situations with tw o beacons

We have verified with simulations that the MLS does not converge on the perpendicular line $\Delta_{l}$ of figure 4 . We have noticed that the estimate converges towards a line through $B_{l}$, but different from the real one [10] (same for $\left.\Delta_{2}\right)$.

In [12], Boley and Sutherland presented a localization system which uses two azimuth angles. Their estimation method is based on the use of "recursive total least squares" and relies on the hypothesis that the robot tracks a straight line. It turns out that their observer does not converge either on $\Delta_{l}$ and $\Delta_{2}$. More generally as proved in [12], their least squares problem does not yield a unique solution for any straight line through $B 1$ or $B 2$. On the contrary, we have been able to check symbolically that the states defined by other straight lines through $B_{1}$ or $B_{2}$ satisfy the rank condition for non-zero linear speeds. Convergence of the filter has been confirmed by simulations.

In the case of a vehicle moving along $\Delta_{3}$, the observations do not depend on the state; the Lie derivatives are all equal to zero. State observation is clearly impossible. 


\section{V.REAL OUTDOOR EXPERIMENTS1}

Real experiments have been run on an outdoor test-track marked out with three beacons with a threewheeled vehicle (figure 5). Reference points have been located by surveyors, in a local frame. White strings can be stretched out on the grass to materialize straight lines or circles of known equations. The principle of our experiments is to make the robot track those reference paths using a CCD camera (see the schematic representation of figure 5), and to compare the MLS estimation results with reality.
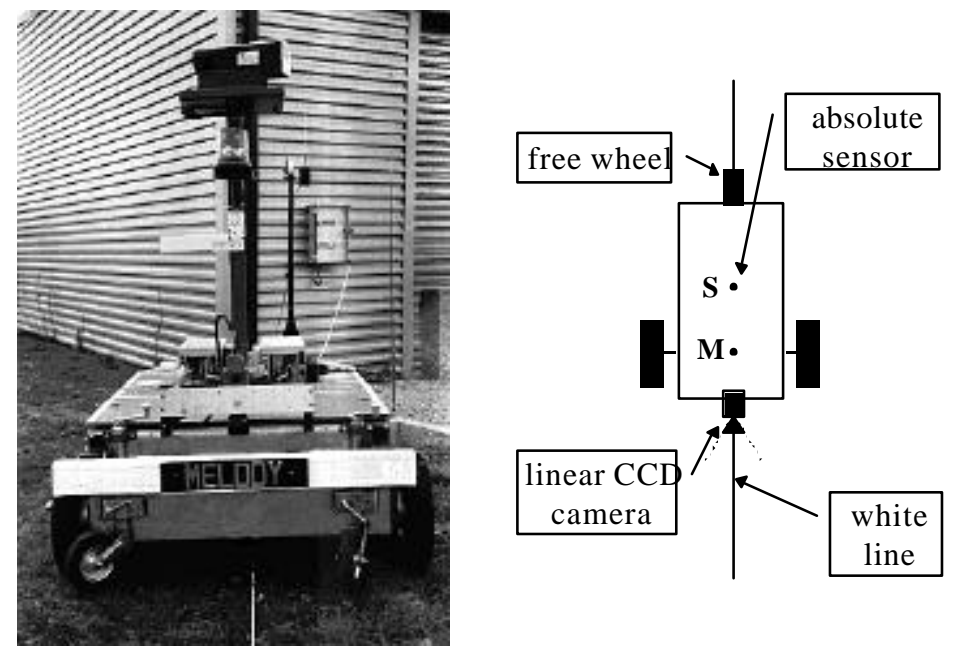

\section{figure 5: the experimental robot "M elody" and the white line}

Thanks to a real-time executive, periodic readings of the encoders, computation of the asynchronous azimuth angles from the CCD camera, on and off switching of the beacons, and all the computations of the estimation process, are done in real time. In the worst case (i.e. when a filtering phase occurs), the MLS needs to compute 1364 floating point operations in $50 \mathrm{~ms}$. This can be done in real-time with a standard 486-based PC system.

\section{A. Straight line experiment}

In the first test, the robot tracks a straight line (speed $\approx 0.2 \mathrm{~m} / \mathrm{s}$ ) in a $40 \mathrm{~m} \times 30 \mathrm{~m}$ field. Before the robot moves, the filter is initialized using a deterministic iterative method. Alternatively, as suggested by Boley and Sutherland, we could use the solution presented in [12], which allows to initialize the filter "on the fly".

\footnotetext{
1 The experimental data used in the paper, together with the executable post-processing version of the filter and Matlab files for visualization are available through anonymous ftp at "ftp://ftp.ec-nantes.fr/Recherche/Garcia".
} 

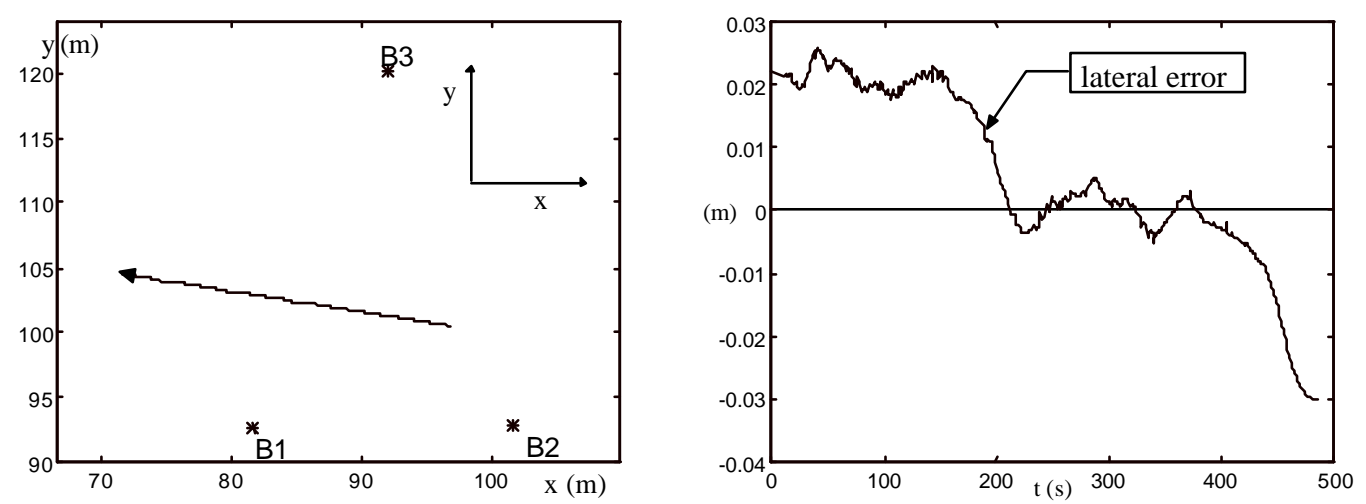

figure 6: experimental path and lateral error

One should keep in mind that the accuracy of the reference marks and beacons positioning is about one centimeter. At the end of the trajectory, the accuracy deterioration is due to the fact that the beacons are all behind the vehicle, hence, in a bad configuration (see figure 6). Additionally, they are distant from the mobile $(\approx 20-35 \mathrm{~m})$, so that one is too far to be detected. Finally, because the sensor is about $1.9 \mathrm{~m}$ above the ground (see figure 5), one degree of roll angle generates a three-centimeter lateral error (our test-track is a lawn and not perfectly planar).

The MLS has a good behavior since the absolute errors remain within two estimated standard deviations, as shown in figure 7. By performing the same path several times, we have checked that the repeatability of the lateral error is smaller than one centimeter (figure 7).
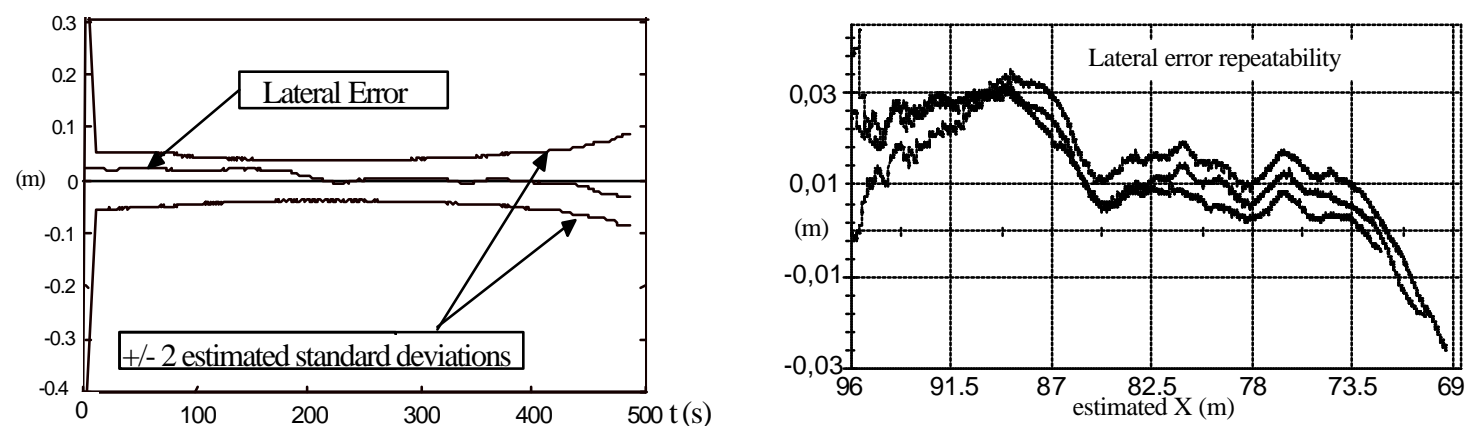

figure 7: lateral error and +/- 2 standard deviations and repeatability

Considering these results, it is clear that the lateral error is not a purely random signal due to measurement noise only. Most probably, it is largely due to the terrain generating the same roll angle at each test. Except for the short transient phase which corresponds to the effect of discrepancies between initial positioning errors, the three curves fit in a narrow one-centimeter wide region. One centimeter is probably closer to the standard deviation of the real lateral error, but this requires testing with a more appropriate set-up. 


\section{B. Circle path}

The second experiment corresponds to the circle of figure 8 . We here compute the signed error distance to the circle.
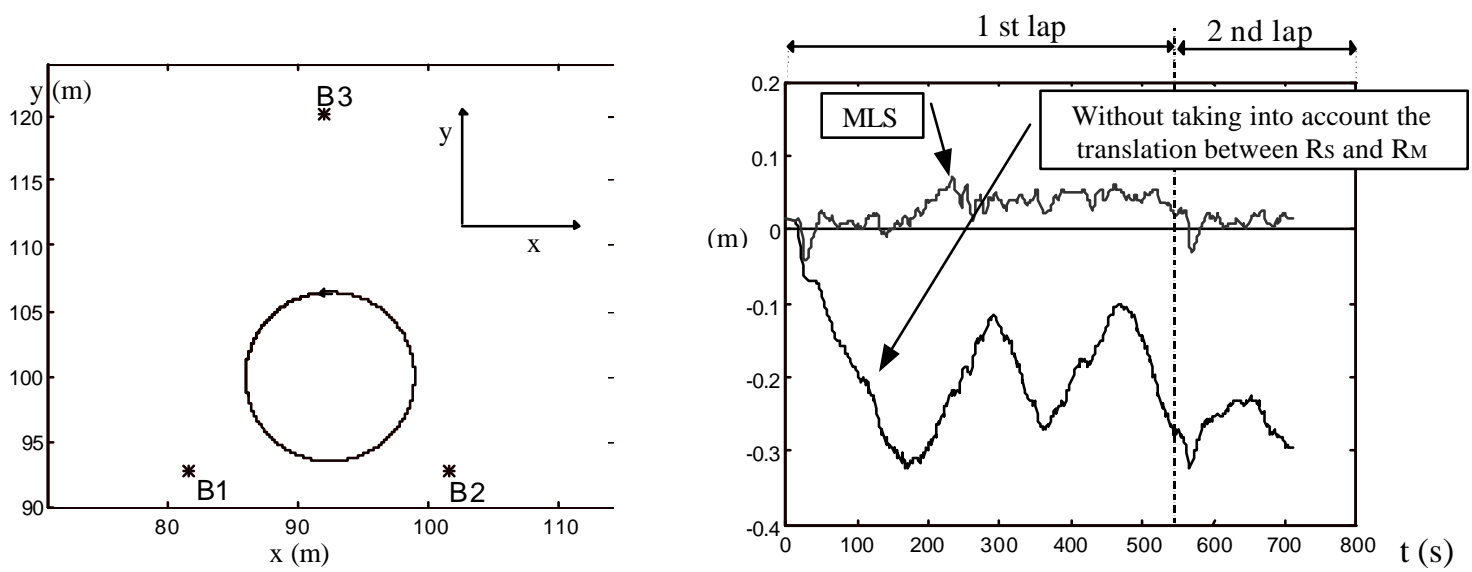

figure 8: experimental path and signed distance to the circle

On figure 8, if we compare the error of the MLS during the first and second lap, the same behavior appears. This phenomenon, like the repeatability of the lateral error (figure 7), is most probably a consequence of the ground and of the string positioning. Notice that a filter which would not consider the translation between $R_{S}$ and $R_{M}$, would have $30 \mathrm{~cm}$ errors.

\section{Test on a degenerate situation}

We have performed a test on the problematic situation with three beacons (see section IV.C.2). We have put the camera of the robot as close as possible to the circle defined by the three beacons.
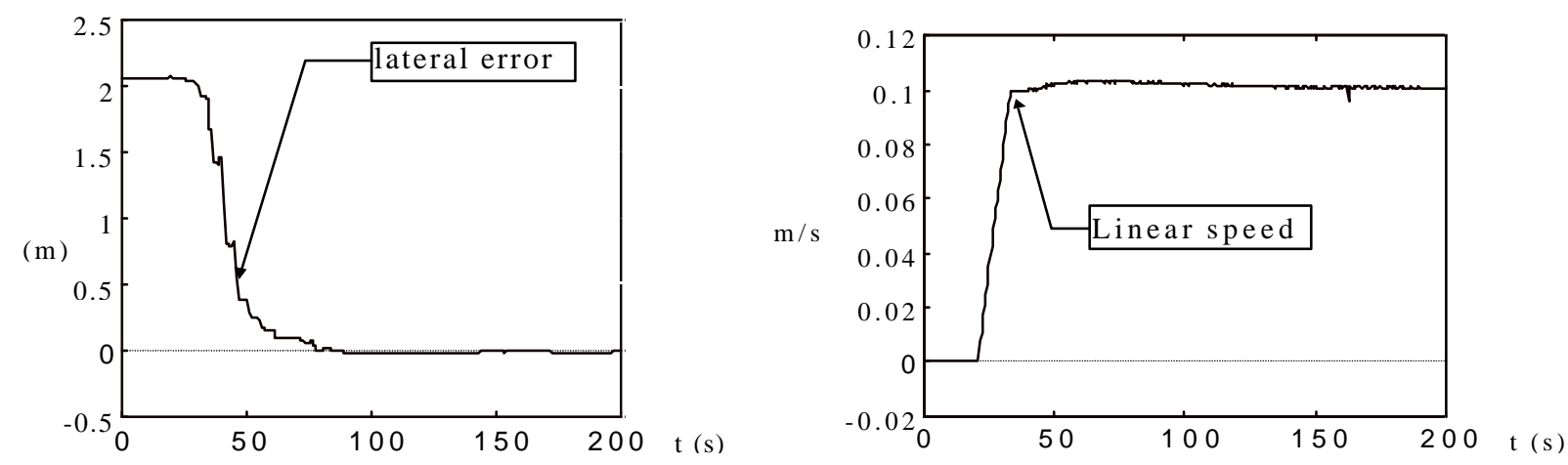

figure 9: lateral error, heading error and estimated linear speed

The robot is initially motionless. Using he first three azimuth angles, the system computes its static localization. The geometrical analysis of part 1 explains why the static localization does not converge toward the real position. Later determinations of the posture of the robot $(t>0)$ are performed by the MLS. 
On figure 9, we can see that, while the robot is motionless, the filter is not able to correct the (large) initial estimation error. But when the robot starts moving ( $(\approx 30 \mathrm{~s})$, the robot leaves the singular situation and the filter converges. This behavior is perfectly consistent with the observability analysis.

\section{CONCLUSION}

In this paper, we have presented a MLS, based on odometry and goniometry, which takes into account each goniometric measurement individually and asynchronously.

Moreover, our solution puts no constraint on the physical location of the goniometric sensor with respect to the vehicle. Such restriction would not have been acceptable on real vehicles, especially civilengineering machines, which are our main area of application.

Although three beacons are necessary to localize a motionless vehicle, our system is able to work when the vehicle detects only two beacons while moving, except on some degenerate trajectories which have been determined thanks to observability analysis. In practice, this is an interesting feature of our solution since, for instance, another vehicle can hide a landmark accidentally. Moreover, it helps to reduce the number of beacons and hence the cost of the infrastructure. As far as observability results are concerned, we think that, as exemplified by Boley and Sutherland, the problematic situations we highlighted are generic and deserve to be tested for any system based on the same type of measurements.

Our paper also reports the results of real outdoor experiments involving a tire-type mobile robot moving on a fairly large non-planar field. Even if the repeatability of our localizer is roughly one centimeter, the current experimental setup does not allow to assert the precision of our system, since the output errors are in the order of the precision of landmark locations, namely one centimeter. A special test track is necessary for precision tests. Such a facility (called "SESSYL") is available by the LCPC (Laboratoire Central des Ponts et Chaussées), the French institution in charge of research and development in the field of road construction. SESSYL is a robotized head with three degrees of freedom that moves along a calibrated rail. Benches will be performed on SESSYL in the framework of our on-going cooperation with the LCPC.

\section{References}

[1] K. Rintanen, H. Mäkelä, K. Koskinen, J. Puputti, M. Sampo, M. Ojala. "Development of an 
autonomous navigation system for an outdoor vehicle". 2nd IFAC Conf. Int. Autonomous Vehicles. pp. 220-225 Espoo, Finland, June 1995.

[2] J. Borenstein, H.R. Everett, L. Feng, D. Wehe. "Mobile robot positioning sensors and techniques", J. of Robot. Syst. 14 (4), pp. 231-249. 1997.

[3] J.J. Leonard and H.F. Durrant-Whyte. "Mobile robot localization by tracking geometric beacons". IEEE Trans. Robot. Automat. Vol. 7, n³. pp. 376-382. June 1991.

[4] H.F. Durrant-Whyte, E. Bell and P. Avery. "The design of a radar-based navigation system for large outdoor vehicles". IEEE Int. Conf. Robot. Automat. pp. 764-769. Nagoya. April 1995.

[5] T. Nishizawa, A. Ohya and S. Yuta, "An implementation of on-board position estimation for mobile robot", IEEE Int. Conf. Robot. Automat. pp. 395-400 Nagoya, May 1995.

[6] U.D. Hanebeneck, G. Schmidt. "Set theoretical localization of fast mobile robots using an angle measurement technique". IEEE Int. Conf. Robot. Automat. Minneapolis. pp. 1387-1394. April 1996.

[7] $\mathrm{Ph}$. Bonnifait and G. Garcia, "A multisensor localization algorithm and its real-time expe-rimental validation". IEEE Int. Conf. Robot. Automat., pp. 1395-1400. Minneapolis, April 1996.

[8] G. Garcia, Ph. Bonnifait and J.-F Le Corre. "A multisensor fusion localization algorithm with selfcalibration of error-corrupted mobile robot parameters", Int. Conf. Advanced Robot. pp. 391-397. Barcelona, September 1995.

[9] B. F. La Scala, R.R. Bitmead and M. R. James. "Conditions for stability of the extended Kalman Filter and Their Application to the Frequency Tracking Problem". Math. of Control, Signal, and Syst. $\mathrm{N}^{\circ}$ 8. pp. 1-26. 1995.

[10] Ph. Bonnifait and G. Garcia. "On the use of non-linear observability conditions in determining test situations for a mobile robot localizer". World Auto. Congress. Int. Symp. Robot. and Manufacturing, pp. 19-24, Vol. 6. Montpellier, May 1996.

[11] R. Hermann and A.J. Krener, "Nonlinear controllability and observability", IEEE Trans. Automatic Control, vol. AC-22, n 5. pp. 728-740. October 1977.

[12] D. Boley and K.T. Sutherland, "A Rapidly Converging Recursive Method for Mobile Robot Localization ". University of Minnesota, Computer Science technical report TR-96-047, 1996. 